\title{
Production and characterization of a recombinant single-chain antibody against Hantaan virus envelop glycoprotein
}

\author{
Jie Yang • Rui Chen - Junxia Wei • Fanglin Zhang • \\ Yong Zhang • Lintao Jia • Yan Yan • Wen Luo • \\ Yunxin Cao • Libo Yao • Jifeng Sun • Zhikai Xu • \\ Angang Yang
}

Received: 22 July 2009/Revised: 18 November 2009/Accepted: 19 November 2009/Published online: 10 December 2009

(C) The Author(s) 2009. This article is published with open access at Springerlink.com

\begin{abstract}
Hantaan virus (HTNV) is the type of Hantavirus causing hemorrhagic fever with renal syndrome, for which no specific therapeutics are available so far. Cell type-specific internalizing antibodies can be used to deliver therapeutics intracellularly to target cell and thus, have potential application in anti-HTNV infection. To achieve intracellular delivery of therapeutics, it is necessary to obtain antibodies that demonstrate sufficient cell type-specific binding, internalizing, and desired cellular trafficking. Here, we describe the prokaryotic expression, affinity purification, and functional testing of a single-chain $\mathrm{Fv}$ antibody fragment ( $\mathrm{scFv}$ ) against HTNV envelop glycoprotein (GP), an HTNV-specific antigen normally located on the membranes of HTNV-infected cells.
\end{abstract}

Jie Yang, Rui Chen, and Junxia Wei contributed equally to this work.

J. Yang $\cdot$ J. Sun $(\bowtie)$

Department of Nephrology, Tangdu Hospital,

Fourth Military Medical University,

Xi'an, Shaanxi 710038, People's Republic of China

e-mail: jifeng-sun@medmail.com.cn

J. Yang $\cdot$ R. Chen $\cdot$ J. Wei $\cdot$ L. Jia $\cdot$ L. Yao

State Key Laboratory of Cancer Biology,

Department of Biochemistry and Molecular Biology,

Fourth Military Medical University,

Xi'an, Shaanxi 710032, People's Republic of China

F. Zhang $\cdot$ Y. Yan $\cdot$ W. Luo $\cdot$ Z. Xu $(\bowtie)$

Department of Microbiology, Fourth Military Medical University,

Xi'an, Shaanxi 710032, People's Republic of China

e-mail: zhikaixu@fmmu.edu.cn

Y. Zhang $\cdot$ Y. Cao $\cdot$ A. Yang $(\bowtie)$

Department of Immunology, Fourth Military Medical University,

Xi'an, Shaanxi 710032, People's Republic of China

e-mail: agyang@fmmu.edu.cn
This HTNV GP-targeting antibody, scFv3G1, was produced in the cytoplasm of Escherichia coli cells as a soluble protein and was purified by immobilized metal affinity chromatography. The purified $\mathrm{scFv}$ possessed a high specific antigenbinding activity to HTNV GP and HTNV-infected Vero E6 cells and could be internalized into HTNV-infected cells probably through the clathrin-dependent endocytosis pathways similar to that observed with transferrin. Our results showed that the E. coli-produced $\mathrm{scFv}$ had potential applications in targeted and intracellular delivery of therapeutics against HTNV infections.

Keywords Hantavirus · Hantavirus envelop glycoprotein . Single-chain Fv antibody fragment · Intracellular delivery

\section{Introduction}

Hantaviruses, which belong to the family of Bunyaviridae, are composed of a spherical lipid envelop, four viral proteins, and three single-stranded negative-sensed RNA segments (Schmaljohn and Dalrymple 1983). While the small (S [ 2.0 kb]) and medium segments (M [ 3.6 kb]) encode the nucleocapsid protein (NP) and the two envelop glycoproteins (GPs) G1 and G2, respectively, the large segment (L $[\sim 6.0 \mathrm{~kb}]$ ) contains the coding sequence for the viral RNA polymerase (Plyusnin et al. 1996). Hantaviruses can infect humans when virus-contaminated excreta from persistently but asymptomatically infected rodents are inhaled (Schmaljohn and Hjelle 1997). Within the genus Hantavirus, two groups have been identified: New World and Old World Hantaviruses. They not only differ in their geographical distribution but also vary regarding the 
pathology of human infections. Old World Hantaviruses, such as Hantaan, Seoul, Dobrava, and Puumala viruses (Schmaljohn and Hjelle 1997; Sjölander et al. 2002), cause hemorrhagic fever with renal syndrome (HFRS), whereas, New World Hantaviruses, such as Sin Nombre and Andes viruses (Schmaljohn and Hjelle 1997; Meyer and Schmaljohn 2000), are the causative pathogen of Hantavirus pulmonary syndrome (HPS). HFRS is characterized by fever, renal failure, thrombocytopenia, and in severe cases hemorrhagic manifestations with a mortality rate of $2-10 \%$ (Peters et al. 1999), while HPS is featured by severe acute respiratory dysfunction and a mortality rate of approximately 50\% (Nichol et al. 1993; López et al. 1996). More than 100,000 cases of HFRS occur annually worldwide and most are documented in China (Song 1999). Hantaan virus (HTNV), the prototype of the Hantavirus genus, is responsible for numerous cases of HFRS. The antiviral drug ribavirin, which is mainly effective in the early phase of HFRS, is currently under clinical investigation but has not been proven to be sufficient to prevent Hantavirus propagation. Instead, the treatment is restricted to supportive procedures to keep life-threatening symptoms under control (Linderholm and Elgh 2001).

Suppression of pathogenic genes via nucleic acid-based reagents holds great promises as novel therapeutic approach against a wide variety of diseases, including infectious diseases, cancer, and genetic disorders. In this regard, antisense oligonucleotides and more recently, small interfering RNAs have also been used (Corey 2007; Dorsett and Tuschl 2004). However, a major challenge to the development of therapeutic nucleic acid drugs is specific and efficient in vivo delivery to target cells. To enhance the therapeutic efficiency, delivery of these innovative therapeutic agents into the cytosol of target cells is required. Recent studies suggest that specific gene silencing in vivo can be achieved by combining these nucleic acid-based reagents with cell typespecific internalizing antibodies. The antibody-directed therapeutic complex enters target cells through receptor endocytosis and is subsequently released into the cytosol to specifically silence target gene expression. Antibody fragments fused with a small nucleic acid-binding protein and antibody fragmentdirected nanoimmunoliposomes are two examples of effective delivery vehicles in vivo (Liu 2007).

To achieve targeted and intracellular delivery of therapeutic genes, antibodies with well-defined cell type-specific binding and internalizing capacity are required. Recombinant antibody technology now allows researchers to engineer lowcost antibodies with specificity and high binding affinity. Single-chain $\mathrm{Fv}$ antibody fragments ( $\mathrm{scFv}$ ) are polypeptides in which the variable domains of immunoglobulin heavy $\left(\mathrm{V}_{\mathrm{H}}\right)$ and light $\left(\mathrm{V}_{\mathrm{L}}\right)$ chain can be connected via a flexible polypeptide linker (Bird et al. 1988). As a delivery vehicle of therapeutic agents, scFv antibody offers several advantages over monoclonal antibody (MAb), e.g., efficient tissue penetration due to their reduced size $(\sim 30 \mathrm{kDa})$. Small recombinant antibodies can be expressed in Escherichia coli, providing an economic and simple method for large-scale production. Binding affinity and internalization of recombinant antibodies can be improved by mutagenesis in complementarity-determining regions (CDRs) and selection via phage display (Jackson et al. 1995; Yang et al. 1995; Schier et al. 1996) and fusion to protein transduction domain (Joliot and Prochiantz 2004; Lindgren et al. 2000). Therefore, scFvs provide useful tool for targeted and intracellular delivery of therapeutically effective molecules.

Hantavirus envelop GPs are transmembrane proteins, which are normally located on the surface of virus-infected cells (Ogino et al. 2004). Thus, they become a suitable target for antibody-mediated delivery of therapeutics. In this study, the generation of a soluble recombinant HTNV GP antibody, scFv3G1, derived from hybridoma cells and the purification of bacterially expressed $\mathrm{scFv} 3 \mathrm{G} 1$ were described, and the cell type-specific binding and internalizing properties of this single-chain antibody were evaluated.

\section{Materials and methods}

\section{Cells}

The African green monkey kidney epithelial cell-derived Vero E6 cell line (Vero C1008, ATCC CRL 1586) was cultured in Dulbecco's modified Eagle's medium (DMEM, Gibro-BRL), supplemented with $10 \%$ heated inactivated fetal bovine serum (FBS) in a $5 \% \mathrm{CO}_{2}$ humidified incubator.

Hybridoma cell line (clone 3G1) and hybridomas producing anti-HTNV GP monoclonal antibody (MAb3G1) were provided by Dr. ZK Xu (Luo et al. 2003; Hu et al. 2005). The mouse hybridoma cell line $3 \mathrm{G} 1$ was propagated for $\mathrm{scFv}$ construction.

\section{Single-chain Fv antibody fragment construction}

Total RNA was isolated from about $10^{7}$ freshly subcloned hybridoma 3G1 cells using Trizol Reagent (Gibco). The first-strand cDNA was synthesized from total RNA using SuperScript ${ }^{\mathrm{TM}}$ II Reverse Transcriptase and oligo (dT) primer (Invitrogen) according to the manufacturer's protocol. cDNAs encoding the antibody variable domains $\left(\mathrm{V}_{\mathrm{H}}\right.$ and $\left.\mathrm{V}_{\mathrm{L}}\right)$ were amplified by separate polymerase chain reaction (PCR) under standard conditions, performed for 30 cycles, using Taq DNA polymerase (Invitrogen) in a thermocycler (Perkin Elmer, PE2400). The $\mathrm{V}_{\mathrm{H}}$ coding regions were amplified with primers VHRev and VHFor, while the $\mathrm{V}_{\mathrm{L}}$ fragments were obtained by PCR using primers VLRev and VLFor. Primers sequences are listed in Table 1 . The amplified DNAs were cloned into the pGEM-T vector (Promega). The constructs 
Table 1 List of primers used for the generation of synthetic genes encoding scFv3G1

\begin{tabular}{ll}
\hline VHRev & (GC)AGGC(GC)CAGCTGCAG(GC)AGTC(AT)GG \\
VHFor & GGTCCCTTGGCCCC \\
VLRev & GACATT(GC)(AT)G(AT)TGAC(GC)CAGTCTCC \\
VLFor & ACGTTTGATCTCGAGCTTGGTCCC \\
VHRevEcoRI & TTTGAATTCGAGGCGCAGCTGCAGGAGTC \\
VHLinkFor & TCCGCCTGAACCGCCTCCACCTGAGGAGACGGTGACCGTGGTCCC \\
VLLinkRev & GGAGGCGGTTCAGGCGGAGGTGGCTCTGGCGGTGGCGGATCGGACATTGTGATGACGCAGTCT \\
VLForSalI & TTTGTCGACACGTTTGATCTCGAGCT \\
\hline
\end{tabular}

Restriction sites EcoRI and SalI used for cloning the scFv are underlined

were then sequenced, and blast of the resulting cDNA sequences and deduced amine acid sequences was performed using the GenBank database and Kabat database (http://www. antibodyresource.com/antibody-database), respectively.

A synthetic gene encoding scFv3G1 was amplified by "splice-overlapped extension" (Horton et al. 1989). The genes encoding the variable domains were independently modified in an initial PCR amplification using primers VHRevEcoRI and VHLinkFor for the $\mathrm{V}_{\mathrm{H}}$ fragment and VLLinkRev and VLForSalI for the $\mathrm{V}_{\mathrm{L}}$ domain (Table 1). VHLinkFor and VLLinkRev carry overlapping sequences encoding the link peptide $\left(\mathrm{Gly}_{4} \mathrm{Ser}\right)_{3}$, while VHRevEcoRI and VLForSalI introduce EcoRI and SalI restriction sites at the upstream and downstream ends of the PCR product, respectively. This allows cloning of the amplified fragment into the corresponding sites of pET32a vector (Novagen), a prokaryotic expression vector harboring a thioredoxin gene and two groups of polyhistidine sequences to facilitate disulfide bond formation, solubility, and purification of recombinant proteins.

Recombinant $\mathrm{scFv}$ antibody expression and purification

A single clone pET32a-scFv3G1 was used to transform fresh competent $E$. coli BL21 (DE3) strain. Colonies were grown in LB medium supplemented with $100 \mu \mathrm{g} / \mathrm{mL}$ ampicillin at $37^{\circ} \mathrm{C}$ until $\mathrm{OD}_{600}$ reaches approximately $0.4-0.6$. Then, bacteria were induced for production of scFv3G1 with $0.2 \mathrm{mM}$ IPTG, and the temperature was shifted to $30^{\circ} \mathrm{C}$ for $3 \mathrm{~h}$. Bacteria from cultures were centrifuged, and the cytoplasm was extracted after sonication. The recombinant protein scFv3G1 was purified using the His-Bind purification kit (Novagen) according to the manufacturer's instructions. Fractions containing the recombinant protein were pooled, concentrated by ultrafiltration (Millipore Corp.), and stored at $-20^{\circ} \mathrm{C}$ until use. The protein concentration was determined using the BCA assay kit (Pierce) with bovine serum albumin (BSA) as a standard.

SDS-PAGE and Western blot analysis

Proteins were separated on $15 \%$ sodium dodecyl sulfate polyacrylamide gel electrophoresis (SDS-PAGE) gels using
Mini-Protein 3 system (Bio-Rad Laboratories) according to the manufacturer's instructions. Samples were mixed with an equal volume of $2 \times$ Laemmli buffer (S3401, SigmaAldrich) and heated at $95^{\circ} \mathrm{C}$ for $5 \mathrm{~min}$. The sample of $15 \mu \mathrm{L}$ protein (or cell lysate) or $10 \mu \mathrm{L}$ low molecular weight protein marker (Pharmacia) was loaded per lane. The gels were run at $60 \mathrm{~mA}$ for $45 \mathrm{~min}$, and resolved protein samples were visualized by staining with Coomassie brilliant blue R-250 (Sigma-Aldrich). The crude cytoplasmic preparation and the purified protein were transferred to a polyvinylidene difluoride membrane (PVDF; Amersham Biosciences) using semidry Western blot transfer apparatus (Bio-Rad Laboratories). The membranes were blocked with 3\% BSA (Sigma-Aldrich) in PBS supplemented with $0.05 \%[v / v]$ Tween-20 (PBST) for $1 \mathrm{~h}$ at room temperature, then washed five times with PBST, and incubated with mouse anti-HisTag antibody as a primary antibody (Qiagen) at 1:3,000 dilution overnight at $4^{\circ} \mathrm{C}$. Then the membranes were washed again and incubated with HRP-conjugated goat anti-mouse IgG as a second antibody (Abcam) at 1:3,000 dilution in PBST at room temperature for $90 \mathrm{~min}$. After washing with PBST, the reaction product was visualized with enhanced chemiluminescence (Amersham) for 5-10 min at room temperature in the dark. For analysis of immunoreactivity of scFv3G1, HTNV GP or unrelated Hantavirus nucleocapsid protein (HTNV NP) was transferred to a PVDF membrane, incubated with scFv3G1 at $100 \mathrm{nM}$ overnight at $4^{\circ} \mathrm{C}$, and followed by incubation with either HRP-conjugated anti-HisTag antibody (Abcam) or HRP-conjugated goat anti-mouse IgG. MAb3G1 was used as positive control while HBsAgspecific scFv15 antibody acted as negative control (Wen et al. 2007a, b).

\section{Indirect ELISA}

Polystyrene plates (96 wells; Nalge Nunc) were coated overnight at $4^{\circ} \mathrm{C}$ with HTNV GP or unrelated HTNV NP antigen at $1: 10$ dilution in $100 \mu \mathrm{L} \quad 0.1 \mathrm{M}$ carbonatebicarbonate buffer ( $\mathrm{pH}$ 9.6). After three washes with PBST, the wells were blocked with $200 \mu \mathrm{L}$ of $1 \%$ BSA in PBST for $1 \mathrm{~h}$ at room temperature and washed as above. The 
primary antibody solutions were $\mathrm{scFv} 3 \mathrm{G} 1$, MAb3G1, or HBsAg-specific scFv15 twofold serially diluted in PBST from 1,000 to $0.1 \mathrm{nM}$. The plates were incubated with primary antibody solutions overnight at $4{ }^{\circ} \mathrm{C}$. The wells were washed again, and the bound antibodies were detected using either HRP-conjugated anti-His-Tag antibody or HRP-conjugated goat anti-mouse IgG in PBST at room temperature for $90 \mathrm{~min}$. After final washing, $100 \mu \mathrm{L}$ of substrate solution composed of $4 \mathrm{mg} o$-phenylenediamine (Sigma-Aldrich) in $10 \mathrm{~mL}$ of $0.1 \mathrm{M}$ citrate-phosphate buffer (pH 5.0) containing $0.01 \% \mathrm{H}_{2} \mathrm{O}_{2}$ was added to each well. The color reaction was developed in the dark for $15 \mathrm{~min}$, and absorbance at $450 \mathrm{~nm}$ was determined using Tecan GENios microplate reader. Assay results are expressed as the means \pm SD of three independent experiments.

Preparation of infected cells

Cultures of Vero E6 cells were inoculated with HTNV strain $76-118$ at a MOI of 0.5 in serum-free medium. After incubation for $1 \mathrm{~h}$ at $37^{\circ} \mathrm{C}$, unbound viruses were removed by a triple washing. Maintenance medium (DMEM with $2 \%$ FBS) was added to the cells and changed every 5 days. The cells were ready for further use 10 days after inoculation. Biosafety level 3 facilities were used throughout these experiments for Hantavirus cultivation.

\section{Flow cytometry}

Flow cytometric analysis was used to evaluate whether scFv3G1 can bind HTNV GP expressed on the cell membrane. The 10-day infected Vero E6 cells or uninfected cells were washed, scraped, and incubated with the recombinant $\mathrm{scFv} 3 \mathrm{G} 1$ at $100 \mathrm{nM}$ in $\mathrm{PBST}$ at $4^{\circ} \mathrm{C}$ for 30 min. In parallel experiments, MAb3G1 was used as positive control, while HBsAg-specific scFv15 antibody acted as negative control. Cells were washed again and stained with fluorescein isothiocyanate (FITC)-conjugated anti-His-Tag antibody (Abcam) or FITC-conjugated goat anti-mouse IgG antibody (Santa Cruz Biotechnology) at 1:1,000 dilution in PBST at $4^{\circ} \mathrm{C}$. After $30 \mathrm{~min}$ of staining, the cells were washed a third time and then analyzed by flow cytometry using a FACSCalibur (Becton Dickinson) instrument.

\section{Immunofluorescence staining}

The immunofluorescence staining was performed to determine whether the scFv3G1 can be internalized into HTNVinfected cells. The Vero E6 cells infected with HTNV for 10 days were seeded on 24 -well coverslips at $3 \times 10^{4}$ cells per well and grown in DMEM (2\% FBS) at $37^{\circ} \mathrm{C}$ in $5 \%$ $\mathrm{CO}_{2}$ incubator overnight. The cells were washed three times in PBS and incubated with FITC-tagged scFv3G1, FITC-tagged MAb3G1, or together with Texas red-tagged transferrin (Molecular Probes) for $30 \mathrm{~min}$ at $37^{\circ} \mathrm{C}$. Then, cells were washed and fixed in 3\% paraformaldehyde for 5 min, followed by DAPI counterstaining for nuclei. After being washed with PBS, the coverslips were mounted on slides with ProLong Gold antifade reagent (Molecular Probes) and observed with a laser scanning confocal microscope (Olympus FV-1000).

\section{Accession numbers}

The nucleotide sequence for $\mathrm{V}_{\mathrm{H}}$ domains of the MAb3G1 is deposited at GenBank as FJ751231 and that for $\mathrm{V}_{\mathrm{L}}$ domains of the MAb3G1 as FJ751232.

\section{Results}

Cloning of the scFv3G1 coding sequence

In order to investigate the cell type-specific binding and internalizing properties by HTNV-infected cells, coding sequences of $\mathrm{V}_{\mathrm{H}}$ and $\mathrm{V}_{\mathrm{L}}$ domains of the MAb3G1 were cloned initially from the parental hybridoma cell line and sequenced. The deduced amino acid sequences were compared with those of variable regions of immunoglobulins stored in the immunological Kabat database. The three loops corresponding to the CDRs of the $\mathrm{V}_{\mathrm{H}}$ and $\mathrm{V}_{\mathrm{L}}$ regions and the amino acid residues involved in the canonical structures were identified. The cDNA sequences of $\mathrm{V}_{\mathrm{H}}$ and $\mathrm{V}_{\mathrm{L}}$ are submitted to the GenBank database (accession number FJ751231 and FJ751232). The cDNAs encoding for MAb3G1 $V_{H}$ and $V_{L}$ were fused by splice-overlapped extension. PCR amplification using primers VHRevEcoRI and VLForSall generated a 0.7-kb fragment (Fig. 1a). This PCR fragment was inserted between EcoRI/SalI cloning site in the pET32a vector and sequenced. Nucleotide and deduced amino acid sequences of scFv3G1 are shown in Fig. 1b.

\section{Production and purification of the scFv3G1}

The recombinant protein was expressed by E. coli BL21 (DE3) strain transformed with pET32a-scFv3G1. Briefly, transformed BL21 cells were grown in LB medium at $37^{\circ} \mathrm{C}$ and incubated with $0.2 \mathrm{mM}$ IPTG at $30^{\circ} \mathrm{C}$ for $3 \mathrm{~h}$. Cytoplasmic recombinant protein, containing a thioredoxin $(11 \mathrm{kDa})$ at the $\mathrm{N}$ terminus and two group of polyhistidines, was extracted after sonication. Immobilized metal affinity purification of scFv3G1 was carried out using Ni-NTA His•Bind Resin that specifically binds the polyhistidine. Eluted fractions were collected and concentrated by ultrafiltration. 
Fig. 1 Construction of HTNV GPs targeting scFv3G1. a PCR for the construction of $\mathrm{scFv}$ antibody-encoding gene. The genes encoding the variable heavy chain $\left(\mathrm{V}_{\mathrm{H}}\right)$ and variable light chain $\left(\mathrm{V}_{\mathrm{L}}\right)$ fragments were amplified and assembled via a short nucleotide linker in a $\mathrm{V}_{\mathrm{H}}$-linker- $\mathrm{V}_{\mathrm{L}}$ format. The amplified products were resolved in $1.5 \%$ agarose gel and stained with ethidium bromide. DNA markers are given in base pairs indicated at the left. b Nucleotide and deduced amino acid sequences of scFv3G1. The linker fragment (Gly4Ser)3 is shown in italics. The complementaritydetermining regions (CDRs) of the $\mathrm{V}_{\mathrm{H}}(H-C D R)$ and $\mathrm{V}_{\mathrm{L}}$ $(L-C D R)$ domains are underlined, and the sequences were identical to those reported in the Kabat database. The four conserved Cys are indicated by stars a

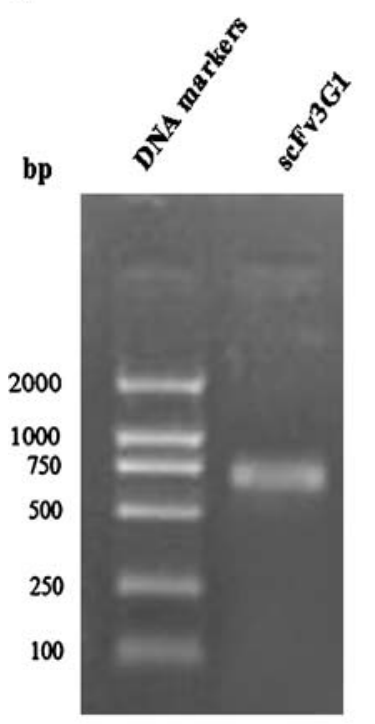

$b^{g}$ aggcgcagctgcaggagtctggggaagtttagtacagcctggagggtccctgaaactc E A Q L Q E S G E G L V Q P G G S L K L

tcctgtgcagcctctggattcactttcagtagttat agtatgtcttgggttcgccagact $\begin{array}{llllllllllllllllllll}S & C^{\prime} & \& & \& & S & G & F & T & F & S & S & Y & S & \text { I } & S & V & V & R & Q & I\end{array}$ -CDRl

$c c$ agagaagaggctggactgggtcgcat ac att agt aat ggtggtggtagcgcctct at

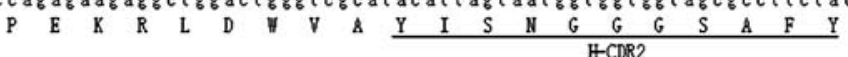

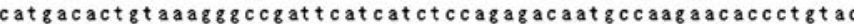
$\begin{array}{lllllllllllllllllllllll}H & D & I & V & K & G & R & F & I & I & S & R & D & N & \& & K & N & I & L & Y\end{array}$

ct aca a at gaccagtctgaagtctgag gacacggccatt tatt actgtgcacgtcagaca $\begin{array}{llllllllllllllllllll}L & Q & I I & I & S & L & K & S & E & D & I & A & I & Y & Y & C^{\prime} & A & R & Q & I\end{array}$

gtctat $g$ at $g g t$ tact actttacttact $g g z g c c a a g z g a c c a c g z t c a c c g t c t c c t c a$ $\begin{array}{lllllllllllllllllllll}V & Y & D & G & Y & Y & F & I & Y & & & G & Q & G & I & I & V & I & V & S & S\end{array}$ $\mathrm{H}-\mathrm{CDR} 3$

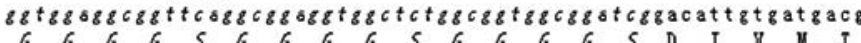
cagtctcc caattcatgtccacatcagtcggagagaggtcagcttcacctgcaaggcc

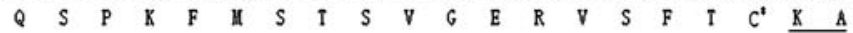

agtcaggatgtgggtactgctgttgcctggtatcaacagaaaccaggacgatctcct a a a $\begin{array}{llllllllllllllllllll}S & Q & D & \nabla & G & I & A & V & \& & Y & Y & Q & Q & K & P & G & R & S & P & K\end{array}$ ct actgat t actgggcatcttcc ggcacactggagtccctgat cgcttcacaggcagt

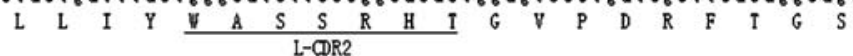
$g g$ atct $g g g$ ac a $g c t t t c a c t c t c a c c a t t a a c a g t g t g c a g t c t g a a g$ act $g g c a g$ at

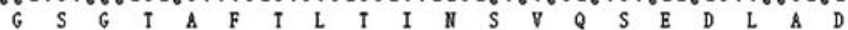

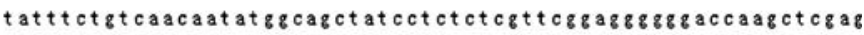
$\begin{array}{llllllllllllllllllll}Y & F & C^{\prime} & Q & Q & Y & G & S & Y & P & \text { L } & S & F & G & G & G & I & K & L & E\end{array}$ at caa acgt

I $\quad \mathbb{K} \quad \mathbf{R}$
The SDS-PAGE of crude cytoplasmic preparation and purified recombinant protein showed a prominent band at the predicted size for scFv3G1 fusion protein molecular weight of about $46 \mathrm{kDa}$ (Fig. 2a), which was detected in all fractions from induced bacteria culture. This His-tagged protein was further examined by Western blot using antiHis-Tag antibody (Fig. 2b). These results showed that this recombinant scFv3G1 protein was released into cytoplasm as a soluble protein, and no degradation products were revealed, suggesting the high solubility and stability of the recombinant protein. The average concentration of affinitypurified protein was $1 \mathrm{mg} / \mathrm{mL}$. In total, about $8 \mathrm{mg}$ of soluble recombinant protein scFv3G1 with more than $90 \%$ purity was obtained per $1 \mathrm{~L}$ of $E$. coli culture.

scFv3G1 specifically recognizes target antigen and retains the antigen-binding activity

In order to investigate the antigen-specific binding of the recombinant scFv3G1, a solid-phase assay was performed using coated plates with HTNV GP as a target antigen. Within the rang tested, increased concentrations of scFv3G1 corresponded to enhanced colorimetric signals, while no significant background was detected in wells coated with the control HTNV NP antigen (Fig. 3a). Moreover, the scFv3G1 showed clear reactivity similar to the parental MAb3G1 (Fig. 3b). The observed difference in binding presumably shows reduced avidity of $\mathrm{scFv}$, based a
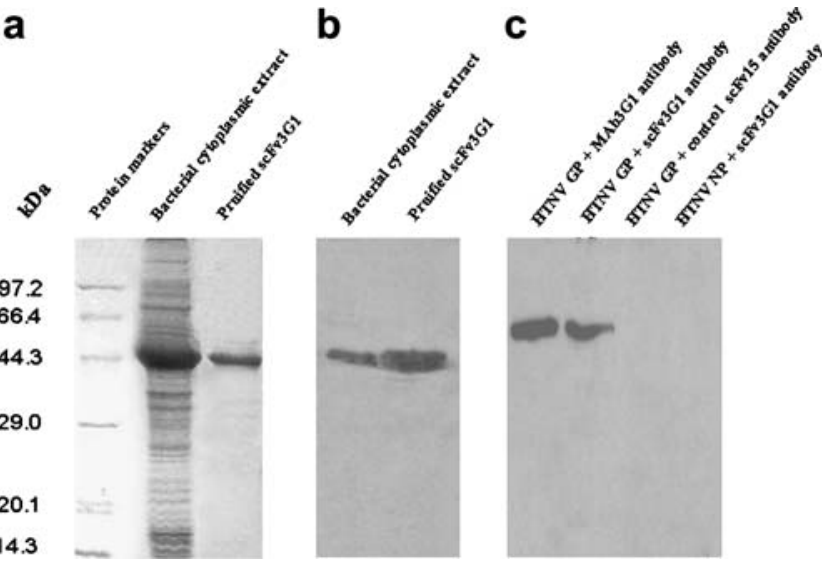

Fig. 2 Expression and purification analysis. Escherichia coli BL21 (DE3) was transformed with the pET32a-scFv3G1 construct. After induction, cytoplasmic proteins were extracted following sonication. Cytoplasmic fraction was purified by immobilized metal affinity chromatography. Bacterial cytoplasmic extract and purified recombinant scFv3G1 antibody were subjected to $15 \%$ SDS-PAGE, stained with Coomassie brilliant blue (a) or transferred to PVDF membrane for Western blot analysis using anti-His-Tag antibody (b). c Western blot analysis of immunoreactivity of scFv3G1. HTNV GP or unrelated HTNV NP was transferred to PVDF membrane, incubated with scFv3G1, and followed by incubation with HRP-conjugated anti-HisTag antibody. Molecular weight markers are given in kilodaltons indicated on the left 
a

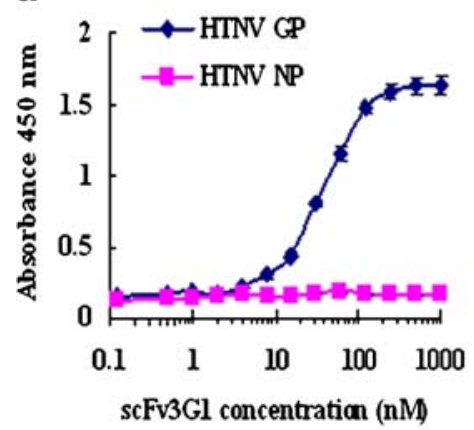

b

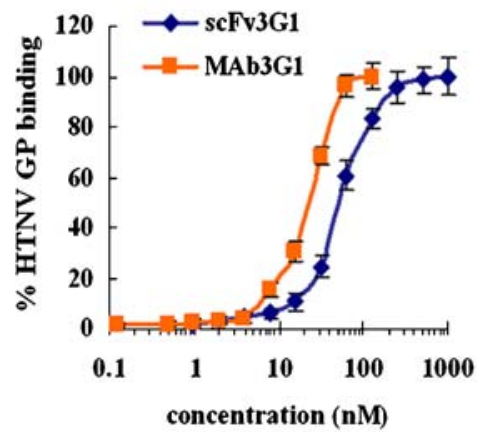

C
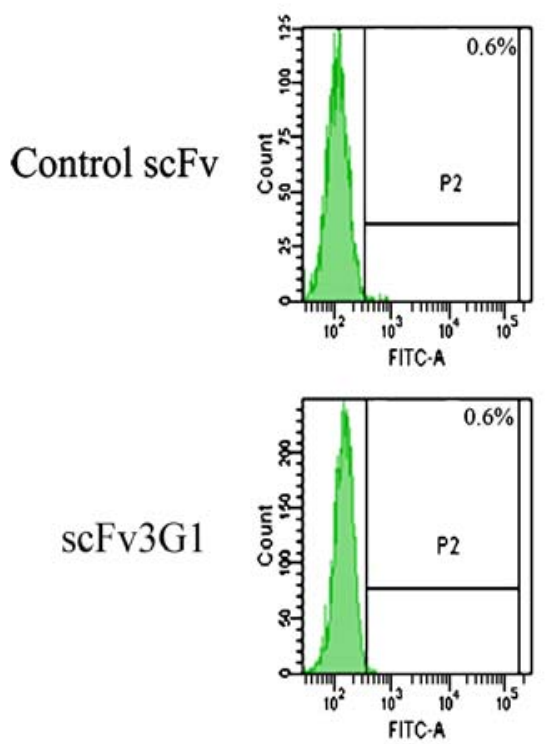

scFv3G1

MAb3G1
Uninfected cells HTNV-infected cells (10d)
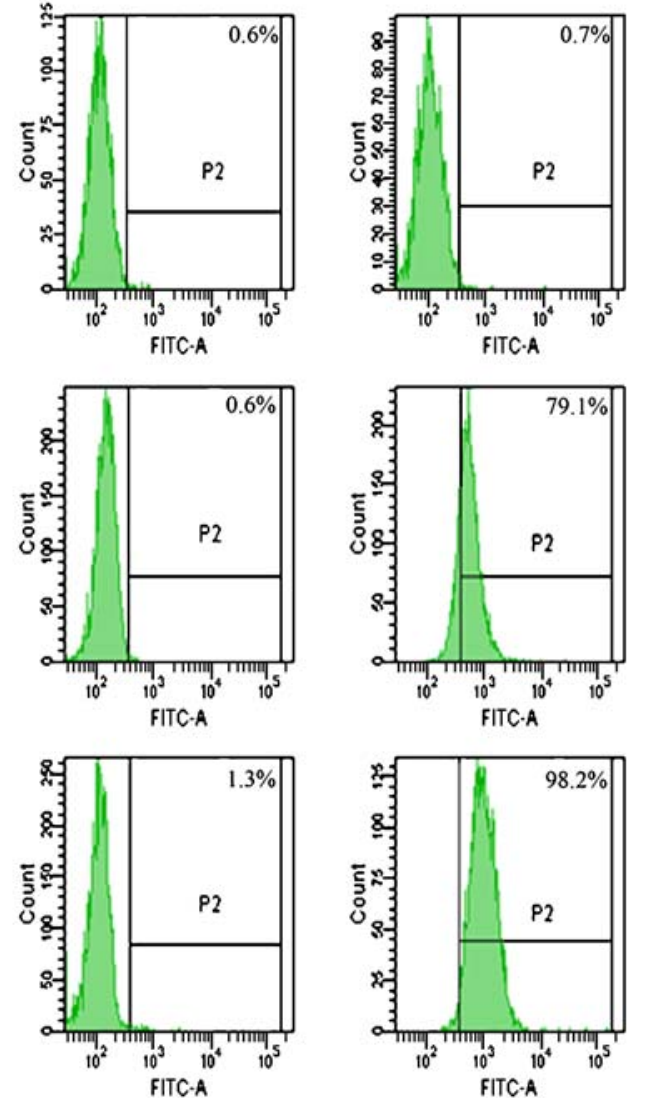

Fig. 3 HTNV GP antigen-specific binding characteristics of scFv3G1. a Solid-phase binding of purified scFv3G1 to HTNV GP antigen as measured by ELISA. The wells were coated with HTNV GP or HTNV NP and then incubated with purified twofold serially diluted scFv3G1 from 1,000 to $0.1 \mathrm{nM}$. b To compare the HTNV GPspecific antigen-binding activity of scFv3G1 to that of MAb3G1, serially diluted scFv3G1 and MAb3G1 were assayed. Results are

on the fact that MAb3G1 is an IgG possessing two identical antigen recognition surface, while scFv3G1 retains only one. We assayed the immunoreactivity of scFv3G1 by Western blot and found that $\mathrm{scFv} 3 \mathrm{G} 1$ detected a polypeptide band of about $56 \mathrm{kDa}$ corresponding to HTNV glycoprotein G2 molecular weight similar to that observed with MAb3G1, while no staining background was detected with unrelated HTNV NP antigen and when irrelevant control HBsAgspecific scFv15 was used instead of scFv3G1 (Fig. 2c). To evaluate whether the scFv3G1 was able to bind HTNV GP on the cell membrane, Vero E6 cells infected with HTNV for 10 days were incubated with the purified scFv3G1 and examined by flow cytometry. This scFv bound with HTNV GP expressed on the membrane of HTNV-infected cells similar to that observed with MAb3G1 (Fig. 3c), while scFv3G1 did not react with uninfected cells, and several other cell lines (data not shown) and control HBsAg-specific scFv15 did not react with HTNV-infected cells in parallel

plotted as percentages of HTNV GP binding. c Flow cytometric analysis to evaluate whether scFv3G1 can bind HTNV GP antigen expressed on the cell membrane. Ten-day HTNV-infected Vero E6 cells or uninfected cells were incubated with $100 \mathrm{nM}$ purified scFv3G1 and followed by detection with FITC-conjugated anti-HisTag antibody

experiments (Fig. 3c). These results indicate that this recombinant scFv3G1 possesses biological activity of HTNV GP-specific binding.

scFv3G1 can be internalized into cytoplasm of HTNV-infected cells

Since intracellular delivery requires a cell type-specific internalizing antibody, we analyzed whether this scFv can be internalized into infected cells. Vero E6 cells preinfected with HTNV were incubated with FITC-tagged scFv3G1 antibody or FITC-tagged MAb3G1 for 30 min. As shown in Fig. 4a, specific fluorescence was observed both on the membrane and in the cytoplasm of HTNV-infected cells. This fluorescence diffusion pattern indicates that both the scFv3G1 and the parental MAb3G1 can be efficiently internalized into the cytoplasm following binding to its target antigen HTNV GP on the surface of HTNV-infected cells. 
Fig. 4 Internalization of scFv3G1. Vero E6 cells infected with HTNV for 10 days were incubated with FITC-labeled scFv3G1 or FITC-labeled MAb3G1 in the absence (a) and in the presence (b) of Texas red-labeled transferrin as an indicator of clathrin-dependent endocytosis at $37^{\circ} \mathrm{C}$ for $30 \mathrm{~min}$ to allow internalization. Nuclei were counterstained with DAPI. Immunofluorescence was observed under confocal microscopy. The colocalization of the scFv3G1 antibody with clathrin-coated endocytic vesicles are indicated by arrows. Scale bar $20 \mu \mathrm{m}$
Uninfected cells

a
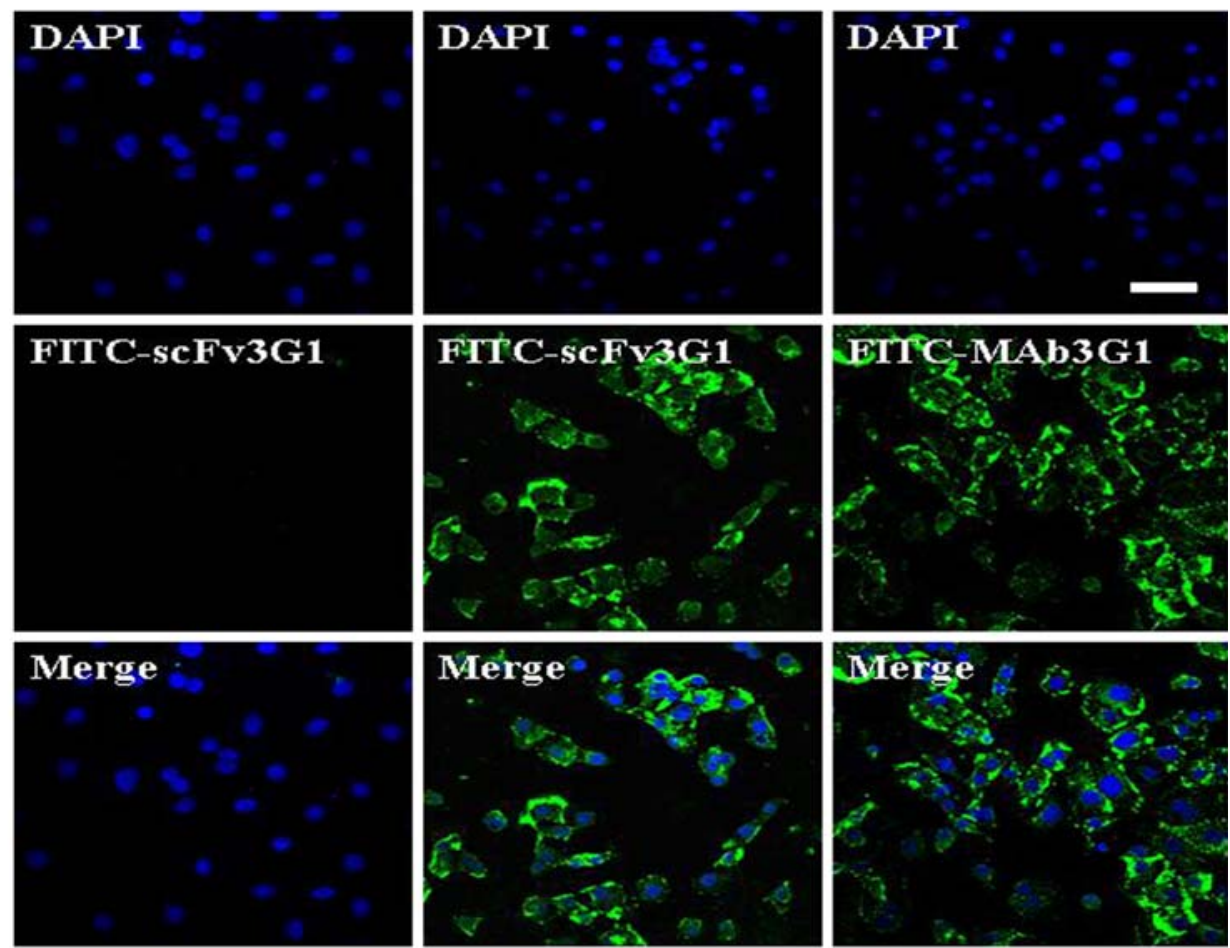

b
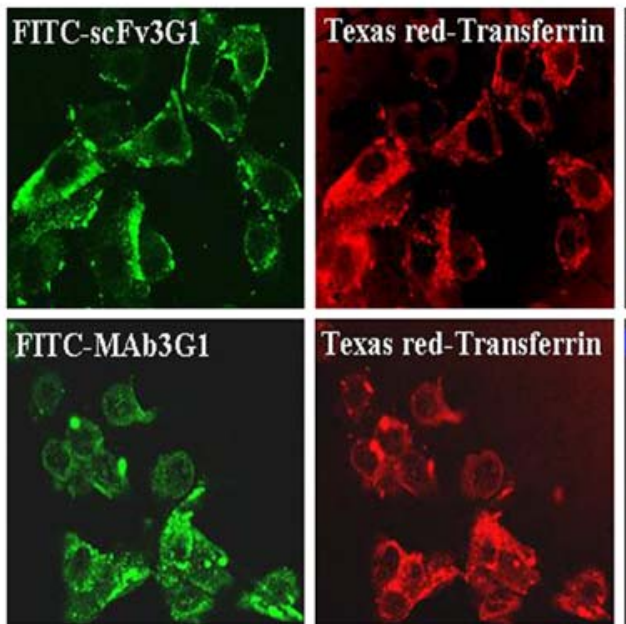
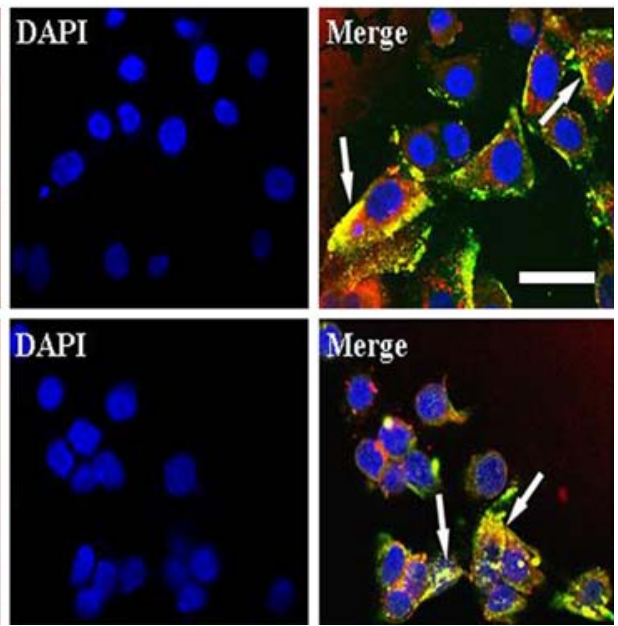

scFv3G1 internalization is mediated by a clathrin-dependent endocytotic pathway

To further investigate the internalizing pathway of this scFv, Vero E6 cells preinfected with HTNV were incubated with FITC-tagged scFv3G1 and Texas red-tagged transferrin, as an indicator of clathrin-dependent endocytosis. As shown in Fig. 4b, merged staining of scFv3G1 and transferrin revealed a colocalization of the recombinant antibody with clathrin-coated endocytic vesicles. Thus, the internalization of $\mathrm{scFv} 3 \mathrm{G} 1$ is likely achieved through clathrin-dependent endocytosis. Taken together, these results suggest that the recombinant single-chain antibody, scFv3G1, possesses the HTNV GP-specific binding activity, and subsequent internalization through receptor endocytosis may allow targeted and intracellular delivery of therapeutics against HTNV infection.

\section{Discussion}

Hantaviruses are enveloped, negative-sense RNA viruses, which can cause human infection after inhalation of aerosols (feces, saliva, and urine) from rodent hosts, and 
are responsible for the occurrence of HFRS and HPS. Although Hantaviruses have been known to infect humans for almost a century, no specific therapeutics are available for the treatment of HFRS and HPS. Recent studies suggest that cell type-specific internalizing antibodies delivery of therapeutic genes is potentially a powerful strategy, given that a direct attack of the infected cells results in the blockade of in vivo viral propagation and even the elimination of the virus. In particular, chimeric proteins, which combine the nucleic acid-binding properties of the small basic protein, protamine, with the specific ligandrecognition properties of antibodies, have been engineered to deliver nucleic acid drugs to HIV-infected cells, HER2 over-expressing cancers (Song et al. 2005), activated LFA1-expressing cells (Peer et al. 2007), and HBV-infected cells (Wen et al. 2007a, b) in cell culture and animal models. Antibody fragments fused to lipids also have been used for effective retargeting of liposome-encapsulated DNAs in cancer therapy (Pirollo et al. 2006, 2007; Brignole et al. 2004). Taken together, these results indicate the potential utility of antibody-targeted system for intracellular trafficking of innovative therapeutic agents.

Hantavirus infection is mediated by the interaction of viral surface GPs with integrin $\alpha_{v} \beta_{3}$ expressed on the host cell surface and subsequent endocytosis (Cheresh 1987; Hynes 1992). Hantavirus GPs include two transmembrane proteins, G1 and G2, which result from posttranslational cleavage of a single glycoprotein precursor and form heterodimers in the endoplasmic reticulum during the intracellular assembly of virus particles (Antic et al. 1992). Both G1 and G2 are normally located on the surface and in the cytoplasm of virus-infected cells (Ogino et al. 2004). Featured by the surface expression of these GPs, Hantavirus-infected cells could be targeted by therapeutics containing the corresponding antibodies.

Engineered antibody fragments such as Fabs and scFvs, which have a lower molecular weight while possessing the affinity to the antigen, have been widely used in targeted therapy and drugs delivery. The prokaryotic E. coli expression system, characterized by easy genetic manipulation and scale-up, and relatively short duration between transformation and protein purification, is among the most popular host for recombinant protein production (ArbabiGhahroudi et al. 2005). In the cytoplasm of E. coli, it is preferable to express antibody fragments in soluble and active form, which can be achieved by one of the following fusion protein strategies: (1) fusion to a polypeptide that is highly soluble and (2) fusion to an enzyme that catalyzes disulfide bond formation. The soluble fraction of fusion protein allows disulfide bond formation and proper folding of the native structure in the cytoplasm and increases the possibility of obtaining soluble proteins with preserved biological activity. Fusion thioredoxin to target protein has been widely used to avoid inclusion body formation and obtain active proteins (LaVallie and McCoy 1995; Wen et al. 2007a, b; Moura-da-Silva et al. 1999; Wu et al. 2003). In this study, the scFv3G1 against HTNV GP was expressed by $E$. coli as a soluble fusion protein and was purified using immobilized metal affinity chromatograph. The purified $\mathrm{scFv}$ was proven active and showed specific binding to HTNV GP antigen and then could be internalized into HTNV-infected cells.

Application of targeted and intracellular delivery of effector moieties requires antibodies capable of internalization, i.e., a receptor-mediated endocytosis via a variety of clathrindependent and clathrin-independent endocytic pathways. Our results revealed that the recombinant HTNV GP antibody, scFv3G1, can be internalized following antigen engagement primarily through a clathrin-dependent endocytic pathway similar to that observed with transferrin. Similar clathrindependent internalization has been reported for the $\mathrm{scFv}$ against HBV antigen (Wen et al. 2007a, b). In the clathrindependent endocytotic pathway, internalized molecules are delivered to the early endosome and then trafficked to the late endosome-lysosome pathway (Mellman 1996; Sorkin and Von Zastrow 2002). Therefore, the internalization characteristics of the scFv3G1 were sufficient for the intracellular delivery of therapeutic agents, which thus may be released to the endolysosomal compartment, followed by rapid permeation into the cytosol and nucleus. The successful generation and purification of the Hantavirus glycoprotein antibody, scFv3G1, together with the demonstration that it can be internalized into HTNV-infected cells, suggests potential application of this recombinant antibody in targeted and intracellular delivery of effector moieties against HTNV infection.

Acknowledgments This work was supported by grants from the National Basic Research Program of China (2004CB518805) and the National Natural Science Foundation of China (30872400). We are grateful to Yunqing $\mathrm{Li}$ and Le Niu for confocal microscopy and Mengyuan Shi, Haitao Wang, Weihong Wen, and Tao Wang for technical help.

Open Access This article is distributed under the terms of the Creative Commons Attribution Noncommercial License which permits any noncommercial use, distribution, and reproduction in any medium, provided the original author(s) and source are credited.

\section{References}

Antic D, Wright KE, Kang CY (1992) Maturation of Hantaan virus glycoproteins G1 and G2. Virology 189:324-328

Arbabi-Ghahroudi M, Tanha J, MacKenzie R (2005) Prokaryotic expression of antibodies. Cancer Metastasis Rev 24:501-519

Bird RE, Hardman KD, Jacobson JW, Johnson S, Kaufman BM, Lee SM, Lee T, Pope SH, Riordan GS, Whitlow M (1988) Singlechain antigen-binding proteins. Science 242:423-426 
Brignole C, Pastorino F, Marimpietri D, Pagnan G, Pistorio A, Allen TM, Pistoia V, Ponzoni M (2004) Immune cell-mediated antitumor activities of GD2-targeted liposomal c-myb antisense oligonucleotides containing $\mathrm{CpG}$ motifs. J Natl Cancer Inst 96:1171-1180

Cheresh DA (1987) Human endothelial cells synthesize and express an Arg-Gly-Asp-directed adhesion receptor involved in attachment to fibrinogen and von Willebrand factor. Proc Natl Acad Sci U S A 84:6471-6475

Corey DR (2007) RNA learns from antisense. Nat Chem Biol 3:8-11

Dorsett Y, Tuschl T (2004) siRNAs: applications in functional genomics and potential as therapeutics. Nat Rev Drug Discov 3:318-329

Horton RM, Hunt HD, Ho SN, Pullen JK, Pease LR (1989) Engineering hybrid genes without the use of restriction enzymes: gene splicing by overlap extension. Gene 77:61-68

Hu G, Xu ZK, Zhang FL, Wu XA, Yan Y, Bai WT, Yu L, Wang HT (2005) Expression of recombinant baculovirus carrying chimeric gene of a diabody against hantaan virus in insect cells. Mian Yi Xue Za Zhi. 21(2):84-87

Hynes RO (1992) Integrins: versatility, modulation and signaling in cell adhesion. Cell 69:11-25

Jackson JR, Sathe G, Rosenberg M, Sweet R (1995) In vitro antibody maturation. Improvement of a high affinity, neutralizing antibody against IL-1 beta. J Immunol 154:3310-3319

Joliot A, Prochiantz A (2004) Transduction peptides: from technology to physiology. Nat Cell Biol 6:189-196

LaVallie ER, McCoy JM (1995) Gene fusion expression systems in Escherichia coli. Curr Opin Biotechnol 6:501-506

Linderholm M, Elgh F (2001) Clinical characteristics of hantavirus infections on the Eurasian continent. Curr Top Microbiol Immunol 256:135-151

Lindgren M, Hällbrink M, Prochiantz A, Langel U (2000) Cellpenetrating peptides. Trends Pharmacol Sci 21:99-103

Liu B (2007) Exploring cell type-specific internalizing antibodies for targeted delivery of siRNA. Brief Funct Genomic Proteomic 6:112-119

López N, Padula P, Rossi C, Lázaro ME, Franze-Fernández MT (1996) Genetic identification of a new hantavirus causing severe pulmonary syndrome in Argentina. Virology 220:223-226

Luo W, Yan Y, Xu ZK, Zhang FL, Wu XA, Liu Y, Bai WT, Wang HT (2003) Construction and sequence analysis of single-chain Fv antibody fragment 3G1. Xi An Lian He Da Xue Xue Bao 6(2):5-8

Mellman I (1996) Membranes and sorting. Curr Opin Cell Biol 8:497498

Meyer BJ, Schmaljohn CS (2000) Persistent hantavirus infections: characteristics and mechanisms. Trends Microbiol 8:61-67

Moura-da-Silva AM, Línica A, Della-Casa MS, Kamiguti AS, Ho PL, Crampton JM, Theakston RD (1999) Jararhagin ECD-containing disintegrin domain: expression in Escherichia coli and inhibition of the platelet-collagen interaction. Arch Biochem Biophys 369:295-301

Nichol ST, Spiropoulou CF, Morzunov S, Rollin PE, Ksiazek TG, Feldmann H, Sanchez A, Childs J, Zaki S, Peters CJ (1993) Genetic identification of a hantavirus associated with an outbreak of acute respiratory illness. Science 262:914-917

Ogino M, Yoshimatsu K, Ebihara H, Araki K, Lee BH, Okumura M, Arikawa J (2004) Cell fusion activities of Hantaan virus envelope glycoproteins. J Virol 78:10776-10782
Peer D, Zhu P, Carman CV, Lieberman J, Shimaoka M (2007) Selective gene silencing in activated leukocytes by targeting siRNAs to the integrin lymphocyte function-associated antigen-1. Proc Natl Acad Sci U S A 104:4095-4100

Peters CJ, Simpson GL, Levy H (1999) Spectrum of hantavirus infection: hemorrhagic fever with renal syndrome and hantavirus pulmonary syndrome. Annu Rev Med 50:531-545

Pirollo KF, Zon G, Rait A, Zhou Q, Yu W, Hogrefe R, Chang EH (2006) Tumor-targeting nanoimmunoliposome complex for short interfering RNA delivery. Hum Gene Ther 17:117-124

Pirollo KF, Rait A, Zhou Q, Hwang SH, Dagata JA, Zon G, Hogrefe RI, Palchik G, Chang EH (2007) Materializing the potential of small interfering RNA via a tumor-targeting nanodelivery system. Cancer Res 67:2938-2943

Plyusnin A, Vapalahti O, Vaheri A (1996) Hantaviruses: genome structure, expression and evolution. J Gen Virol 77:2677-2687

Schier R, McCall A, Adams GP, Marshall KW, Merritt H, Yim M, Crawford RS, Weiner LM, Marks C, Marks JD (1996) Isolation of picomolar affinity anti-c-erbB-2 single-chain Fv by molecular evolution of the complementarity determining regions in the center of the antibody binding site. J Mol Biol 263:551-567

Schmaljohn CS, Dalrymple JM (1983) Analysis of Hantaan virus RNA: evidence for a new genus of bunyaviridae. Virology 131:482491

Schmaljohn C, Hjelle B (1997) Hantaviruses: a global disease problem. Emerg Infect Dis 3:95-104

Sjölander KB, Golovljova I, Vasilenko V, Plyusnin A, Lundkvist A (2002) Serological divergence of Dobrava and Saaremaa hantaviruses: evidence for two distinct serotypes. Epidemiol Infect 128:99-103

Song G (1999) Epidemiological progresses of hemorrhagic fever with renal syndrome in China. Chin Med J (Engl) 112:472-477

Song E, Zhu P, Lee SK, Chowdhury D, Kussman S, Dykxhoorn DM, Feng Y, Palliser D, Weiner DB, Shankar P, Marasco WA, Lieberman J (2005) Antibody mediated in vivo delivery of small interfering RNAs via cell-surface receptors. Nat Biotechnol 23:709-717

Sorkin A, Von Zastrow M (2002) Signal transduction and endocytosis: close encounters of many kinds. Nat Rev Mol Cell Biol 3:600 614

Wen WH, Liu JY, Qin WJ, Zhao J, Wang T, Jia LT, Meng YL, Gao H, Xue CF, Jin BQ, Yao LB, Chen SY, Yang AG (2007a) Targeted inhibition of HBV gene expression by single-chain antibody mediated small interfering RNA delivery. Hepatology 46:8494

Wen WH, Qin WJ, Gao H, Zhao J, Jia LT, Liao QH, Meng YL, Jin BQ, Yao LB, Chen SY, Yang AG (2007b) An hepatitis B virus surface antigen specific single chain of variable fragment derived from a natural immune antigen binding fragment phage display library is specifically internalized by HepG2.2.15 cells. J Viral Hepat 14:512-519

Wu SC, Yu CH, Lin CW, Chu IM (2003) The domain III fragment of Japanese encephalitis virus envelope protein: mouse immunogenicity and liposome adjuvanticity. Vaccine 21:2516-2522

Yang WP, Green K, Pinz-Sweeney S, Briones AT, Burton DR, Barbas CF 3rd (1995) CDR walking mutagenesis for the affinity maturation of a potent human anti-HIV-1 antibody into the picomolar range. J Mol Biol 254:392-403 